TRANSACTIONS OF THE

AMERICAN MATHEMATICCAL SOCIETY

\title{
THE EIGENVALUE SPECTRUM AS MODULI FOR FLAT TORI
}

BY

\author{
SCOTT WOLPERT ${ }^{1}$
}

\begin{abstract}
A flat torus $T$ carries a natural Laplace Beltrami operator. It is a conjecture that the spectrum of the Laplace Beltrami operator determines $T$ modulo isometries. We prove that, with the exception of a subvariety in the moduli space of flat tori, this conjecture is true. A description of the subvariety is given.
\end{abstract}

A flat torus $T$ is the Riemannian manifold that is the quotient of $\mathbf{R}^{n}$ by a lattice of maximal rank. $T$ has a Laplace operator and an associated sequence of eigenvalues. The following question arises: To what extent is the geometry of $T$ determined by the eigenvalue spectrum? J. Milnor observed that there exist two nonisometric 16-dimensional flat tori with the same eigenvalue spectrum [1], [2], [7]. We show that this phenomenon is nongeneric in the moduli space $O(n) \backslash \mathrm{GL}(n ; \mathbf{R}) / \mathrm{GL}(n ; \mathrm{Z})$ for flat $n$-dimensional tori. In particular, given tori $\mathbf{R}^{n} / A_{0} \mathbf{Z}^{n}$ and $\mathbf{R}^{n} / A_{1} \mathbf{Z}^{n}$ with the same eigenvalue spectrum, they are either isometric or the quadratic forms $\left(A_{0}^{t} A_{0}\right)$ and $\left(A_{1}^{t} A_{1}\right)$ lie on a subvariety in the space of positive definite quadratic forms. The book of M. Berger, P. Gaudauchon and E. Mazet [1] and article of M. Berger [2] are suggested as general references.

A lattice is a discrete subgroup of $\mathrm{R}^{n}$ and can be prescribed as $A \mathrm{Z}^{n}$ with $A$ a fixed matrix. An $n$-dimensional torus $T$ is $\mathbf{R}^{n}$ factored by a lattice $L=A \mathrm{Z}^{n}$ with $A \in \mathrm{GL}(n ; \mathbf{R})$. The metric structure of $\mathbf{R}^{n}$ projects to $T$ such that volume $(T)=|\operatorname{det} A| ; \quad T$ carries a Laplace Beltrami operator $\Delta=$ $-\Sigma_{i} \partial^{2} / \partial x_{i}^{2}$, the projection of the Laplacian of $\mathbf{R}^{n}$. The set $\tilde{L}=\left\{\tilde{a} \in \mathbf{R}^{n} \mid \tilde{a}^{t} a\right.$ $\in \mathbf{Z}, \forall a \in L\}$ is the dual lattice of $L ; \tilde{L}=\left(A^{-1}\right)^{t} \mathbf{Z}^{n}$. The eigenfunctions of $T$ are $\exp \left(2 \pi i \tilde{a}^{t} x\right)$ for $x \in \mathbf{R}^{n}, \tilde{a} \in \tilde{L}$. The eigenvalues of $T$ are given as $4 \pi^{2}\|\tilde{a}\|^{2}$ for $\tilde{a}$ arbitrary in $\tilde{L}$ where \|\| is the Euclidean norm. The lengths of closed geodesics of $T$ are given as $\|a\|$ for $a$ aribtrary in $L$. The eigenvalues of $T$ determine the dimension, volume and the lengths of closed geodesics of $T$ [1], [2]. Tori $T_{0}$ and $T_{1}$ are called isospectral if they have the same sequence with multiplicities of eigenvalues.

Received by the editors April 14, 1977 and, in revised form, July 25, 1977.

AMS (MOS) subject classifications (1970). Primary 53C99, 58G99; Secondary $10 \mathrm{E} 99$.

Key words and phrases. Flat torus, Laplace Beltrami operator, spectrum, moduli, lattice, quadratic forms.

${ }^{1}$ Research partially supported by the National Science Foundation Grant NPS 75-07403.

- American Mathematical Society 1978 
Let $P$ be a symmetric matrix which defines a quadratic form on $\mathbf{R}^{n}$. The spectrum of $P$ is defined to be the sequence with multiplicities of values $\gamma=P[N]$ where $P[N]=N^{t} P N, N \in \mathbf{Z}^{n}$. The sequence of squares of lengths of closed geodesics of $\mathbf{R}^{n} / A \mathrm{Z}^{n}$ is the spectrum of $A^{t} A=Q$; the sequence of eigenvalues is the spectrum of $4 \pi^{2}\left(A^{-1}\right)\left(A^{-1}\right)^{t}=4 \pi^{2} Q^{-1}$. The Jacobi inversion formula yields for positive $\tau$,

$$
\sum_{N \in Z^{n}} \exp \left(-4 \pi^{2} \tau Q^{-1}[N]\right)=\frac{\operatorname{volume}(T)}{(4 \pi \tau)^{n / 2}} \sum_{M \in Z^{n}} \exp \left(\frac{-1}{4 \tau} Q[M]\right) \text {. }
$$

We now describe the manner in which $O(n) \backslash \mathrm{GL}(n ; \mathbf{R}) / \mathrm{GL}(n ; \mathbf{Z})$ is the moduli space of flat tori. To $A \in \mathrm{GL}(n ; \mathbf{R})$ is associated the lattice $A \mathrm{Z}^{n}$. The tori $\mathbf{R}^{n} / A \mathbf{Z}^{n}$ and $\mathbf{R}^{n} / B \mathbf{Z}^{n}$ are isometric if and only if $A \mathbf{Z}^{n}$ and $B \mathbf{Z}^{n}$ are equivalent by multiplication on the left by an element of $O(n)$, the orthogonal group in $n$-dimensions. The matrices $A$ and $B$ are associated to the same lattice if and only if they are equivalent by multiplication on the right by an element of $\mathrm{GL}(n ; \mathbf{Z})$. The tori $\mathrm{R}^{n} / A \mathrm{Z}^{n}$ and $\mathbf{R}^{n} / B \mathrm{Z}^{n}$ are isometric if and only if $A$ and $B$ are equivalent in $O(n) \backslash \mathrm{GL}(n ; \mathrm{R}) / \mathrm{GL}(n ; \mathrm{Z})$. Denote the space of positive definite symmetric $n \times n$ matrices as $\delta(n ; \mathbf{R})$; we observe that the map

$$
A \in \mathrm{GL}(n ; \mathbf{R}) \rightarrow A^{t} A \in \delta(n ; \mathbf{R})
$$

determines a bijection of $O(n) \backslash \mathrm{GL}(n ; \mathbf{R})$ to $\delta(n ; \mathbf{R})$. Let $e_{i}$ be the $i$ th column of the identity matrix in GL $(n ; \mathbf{R})$ and $e_{i j}=e_{i}+e_{j}$. We consider $\delta(n ; \mathbf{R})$ to be embedded in $\mathbf{R}^{m}$ for $m=n(n+1) / 2$. The cartesian coordinates of $P=\left(p_{i j}\right) \in \delta(n ; \mathbf{R})$ are $P\left[e_{i}\right]=p_{i i}$ and $\left(P\left[e_{i j}\right]-P\left[e_{i}\right]-P\left[e_{j}\right]\right) / 2$ $=p_{i j}$. For later reference we define $E \in\left\{e_{k}, e_{i j} \mid 1<k<n, 1<i<j<n\right\}$.

We now generalize two theorems for Riemann surfaces to $n$-dimensional tori [4], [6].

THEOREM 1. Let $T_{s}$ be a continuous family of isospectral tori defined for $s \in[0,1]$. The tori $T_{s}, s \in[0,1]$, are isometric.

Proof. We lift $T_{s}$ a continuous curve into $O(n) \backslash \mathrm{GL}(n ; \mathbf{R}) / \mathrm{GL}(n ; \mathbf{Z})$ to a curve $g(s)$ of $[0,1]$ into $O(n) \backslash \mathrm{GL}(n ; \mathbf{R})$. Thus $\left(g(s)^{t} g(s)\right)$ is a curve into $\delta(n: \mathbf{R})$. The forms $\left(g(s)^{t} g(s)\right)$ have a spectrum independent of $s$. Thus for every $N \in \mathbf{Z}^{n}$, $\left(g(s)^{t} g(s)\right)[N]$ is a continuous function with range contained in the spectrum of $\left(g(0)^{t} g(0)\right)$. Since the spectrum of an element of $\delta(n ; \mathbf{R})$ is a discrete set, the functions $\left(g(s)^{t} g(s)\right)[N]$ are constant. By the coordinate description of $\delta(n ; \mathbf{R}),\left(g(s)^{t} g(s)\right)$ is constant; thus $g([0,1])$ is a point in $O(n) \backslash \mathrm{GL}(n ; \mathbf{R})$.

The following result is due to $M$. Kneser (unpublished) [1].

THEOREM 2. The total number of nonisometric tori with a given eigenvalue spectrum is finite. 
Proof. By contradiction assume the existence of a sequence of distinct isospectral tori $T_{1}, \ldots, T_{l}, \ldots$. The tori each have the same dimension, volume and length of the shortest closed geodesic. Choose a lattice $L_{l}$ which represents the torus $T_{l}$. By Mahler's compactness theorem a subsequence $L_{k}$ exists which converges to $L_{0}$ (i.e., matrices $A_{k}$ exist with $A_{k} \mathbf{Z}^{n}=L_{k}$ and $A_{k}$ converge to $A_{0}$ where $L_{0}=A_{0} \mathrm{Z}^{n}$ ) [3]. Let $U$ be a neighborhood of $S_{0}=A_{0}^{t} A_{0}$ with $\bar{U}$ compact and $\bar{U} \subset \delta(n ; \mathbf{R})$. Define $c_{1}=\max \{S[e] \mid e \in E, S \in U\}$. Since $S \in \delta(n ; \mathbf{R})$ can be diagonalized by conjugation with an orthogonal matrix, we have for $\lambda_{\min }(S)$ the smallest (resp. $\lambda_{\max }(S)$ the largest) eigenvalue $\lambda_{\min }(S)\|N\|^{2} \leqslant S[N] \leqslant \lambda_{\max }(S)\|N\|^{2}$ for $N \in \mathbf{Z}^{n}$. Now from the inclusion $\bar{U} \subset \delta(n ; \mathbf{R})$ it follows that $\lambda_{\min }(S)>c_{2}>0$ for $S \in U$. In particular, for $N \in \mathbf{Z}^{n},\|N\|^{2}>c_{1} / c_{2}$ and $S \in U$ it follows that $S[N] \geqslant \lambda_{\min }(S)\|N\|^{2}>$ $c_{1}$. Reformulating this we have for $S \in U, e \in E$ and $M \in \mathbf{Z}^{n}$ with $S[M]=$ $S_{0}[e]$ that $M \in F=\left\{N \in \mathbf{Z}^{n} \mid\|N\|^{2}<c_{1} / c_{2}\right\}$. We now consider for $N \in F$ the finite collection of functions $S[N]$ with domain $U$. A neighborhood $V \subset U$ is defined as follows: $V=\left\{S \in U|| S[N]-S_{0}[N]|<| S[N]-\right.$ $S_{0}[M] \mid$ for each $N \in E$ and all $M$ with $\left.S_{0}[N] \neq S_{0}[M]\right\}$. Now for $k$ sufficiently large, $\left(A_{k}^{t} A_{k}\right) \in V$. In particular, for $e \in E, \mid\left(A_{k}^{t} A_{k}\right)[e]-$ $\left(A_{0}^{t} A_{0}\right)[e] \mid$ is strictly less than the distance between $\left(A_{k}^{t} A_{k}\right)[e]$ and any value distinct from $\left(A_{0}^{t} A_{0}\right)[e]$ in the spectrum of $\left(A_{0}^{t} A_{0}\right)$. Noting that $\left(A_{k}^{t} A_{k}\right)$ and $\left(A_{0}^{t} A_{0}\right)$ have the same spectrum we conclude $\left(A_{k}^{t} A_{k}\right)[e]=\left(A_{0}^{t} A_{0}\right)[e]$ for all $e \in E$, the desired contradiction.

The following theorem describes the structure of the equivalence relation, having the same spectrum, for forms.

THEOREM 3. There is a properly discontinuous group $G_{n}$ acting on $\delta(n ; \mathbf{R})$ containing the transformation group induced by the $\mathrm{GL}(n ; \mathrm{Z})$ action $S \rightarrow S[\mathscr{L}]$, $S \in \mathcal{S}(n ; \mathbf{R}), \mathscr{Z} \in \mathrm{GL}(n ; \mathrm{Z})$. Given $P, S \in \mathcal{S}(n ; \mathbf{R})$ with the same spectrum either $g(P)=S$ for some $g \in G_{n}$ or $P, S \in V_{n}$ where $\mathbf{V}_{n}$ is a subvariety of $\delta(n ; \mathbf{R}) . \mathbf{V}_{n}=\{Q \in \mathcal{S}(n ; \mathbf{R}) \mid \operatorname{spec}(Q)=\operatorname{spec}(R), R \in \mathcal{S}(n ; \mathbf{R})$ with $R \neq$ $g(Q)$ for all $\left.g \in G_{n}\right\} . V_{n}$ is the intersection of $\delta(n ; \mathbf{R})$ and a countable union of subspaces of $\mathbf{R}^{m}$.

The proof is initiated with the following lemmas.

Lemma 4. Let $P, S \in \delta(n ; \mathbf{R})$ have the same spectrum. Neighborhoods $U$ of $P, V$ of $S$ and a finite number of maps $g_{1}, \ldots, g_{l}$ with domain $U$ are defined. For $Q \in U$ and $R \in V$ with the same spectrum then $R=g_{j}(Q)$ for some $j$, $1<j<l$. The maps $g_{j}$ are linear in the coordinates of $\mathbf{R}^{m}$ and have rational coefficients.

Proof. Set $c_{1}=2 \max \{S[e] \mid e \in E\}$. We can, noting that $E$ is finite, choose a neighborhood $V$ of $S$ such that for $R \in V, \max \{R[e] \mid e \in E\}<c_{1}$. 
A neighborhood $U_{1}$ of $P$ is chosen with $\lambda_{\min }(Q) \geqslant c_{2}>0$ for $Q \in U_{1}$. Thus considering $\lambda_{\min }$ we have $Q[M]>c_{1}$ for $M \in \mathbf{Z}^{n}, Q \in U_{1}$ with $\|M\|^{2}>$ $c_{1} / c_{2}$. Now let $Q_{0} \in U_{1}$ and $R_{0} \in V$ be such that vectors $M_{k}, M_{i j}$ exist with $Q_{0}\left[M_{k}\right]=R_{0}\left[e_{k}\right], 1<k<n$ and $Q_{0}\left[M_{i j}\right]=R_{0}\left[e_{i j}\right], 1<i<j<n$. A map $R=g(Q)$ linear in the coordinates of $\mathbf{R}^{m}$ is defined by the equations $Q\left[M_{k}\right]=R\left[e_{k}\right], 1<k<n, Q\left[M_{i j}\right]=R\left[e_{i j}\right], 1<i<j<n$. The map $g$ has rational coefficients. Let $G$ be the set of all maps $R=g_{\alpha}(Q), Q \in U_{1}$ with (i) $g_{\alpha}$ defined by equations $R\left[e_{k}\right]=Q\left[M_{k}^{\alpha}\right], M_{k}^{\alpha} \in \mathbf{Z}^{n}, 1<k<n, R\left[e_{i j}\right]=$ $Q\left[M_{i j}^{\alpha}\right], M_{i j}^{\alpha} \in \mathbf{Z}^{n}, 1<i<j<n$; (ii) $g_{\alpha}\left(U_{1}\right) \cap V \neq \varnothing$. Referring to the definitions of $U_{1}$ and $V$ it follows that $\left\|M_{k}^{\alpha}\right\|^{2},\left\|M_{i j}^{\alpha}\right\|^{2}<c_{1} / c_{2}$. Thus $G=$ $\left\{g_{1}, \ldots, g_{l}\right\}$ is finite. We restrict our consideration to those $g_{j}, 1<j<l$, such that a fixed neighborhood $U \subset U_{1}$ of $P$ exists with $g_{j}(U) \subset V$. Now for $Q, R \in \mathcal{S}(n ; \mathbf{R})$ with the same spectrum a bijection $\beta$ of $\mathbf{Z}^{n}$ necessarily exists with $Q[\beta(N)]=R[N]$ for all $N \in \mathbf{Z}^{n}$. Consequently, for $Q \in U$ and $R \in$ $V$ with the same spectrum, $R=g_{j}(Q)$ for some $j, 1 \leqslant j \leqslant l$. The proof is complete.

LEMMA 5. Let $P$ and $S$ have the same spectrum and $\beta$ be the bijection of $Z^{n}$ such that $P[\beta(N)]=S[N]$ for all $N \in Z^{n}$. Let $g$ be the map with domain $U, a$ neighborhood of $P$, defined by $R=g(Q)$ where $Q\left[M_{k}\right]=R\left[e_{k}\right], 1<k<n$, and $Q\left[M_{i j}\right]=R\left[e_{i j}\right], 1<i<j<n$. Assume furthermore that $S=g(P)$. Then either $Q[\beta(N)]=g(Q)[N]$ for all $Q \in \delta(n ; \mathbf{R})$ or $\{Q \in \delta(n ; \mathbf{R}) \mid \operatorname{spec}(Q)$ $=\operatorname{spec}(g(Q)), g(Q) \in \delta(n ; \mathbf{R})\}$ is a subvariety of $\delta(n ; \mathbf{R})$. In the latter case $\{Q \in \delta(n ; \mathbf{R}) \mid \operatorname{spec}(Q)=\operatorname{spec}(g(Q)), g(Q) \in \delta(n ; \mathbf{R})\}$ is the intersection of $\delta(n ; \mathbf{R})$ and a countable union of subspaces of $\mathbf{R}^{m}$.

Proof. It is clear that $g$ is a linear map of $\mathbf{R}^{m}$ to $\mathbf{R}^{m}$. Let $\beta$ be a bijection of $\mathbf{Z}^{n}$; then $\left\{Q \in \mathbf{R}^{m} \mid Q[\beta(N)]=g(Q)[N], N \in \mathbf{Z}^{n}\right\}$ is the intersection of countably many subspaces and thus is itself a subspace. Now either $\mathbf{V}(\beta) \stackrel{\text { def }}{=}\left\{Q \in S(n ; \mathbf{R}) \mid Q[\beta(N)]=g(Q)[N], N \in \mathbf{Z}^{n}\right\}$ equals $\delta(n ; \mathbf{R})$ for some bijection $\beta$, or for every bijection $\beta$ of $Z^{n}, V(\beta)$ is the intersection of $\delta(n ; \mathbf{R})$ and a proper subspace of $\mathbf{R}^{m}$. Reversing the roles of $Q$ and $Q^{-1}$ in the Jacobi inversion formula we observe that $\operatorname{spec}(Q)$ determines $\mid$ det $Q \mid$. The boundary of $\delta(n ; \mathbf{R}) \subset \mathbf{R}^{m}$ consists of matrices of zero determinant. It is thus immediate that for $Q \in \delta(n ; \mathbf{R})$ with $\operatorname{spec}(Q)=\operatorname{spec}(g(Q))$ that $g(Q) \in$ $\delta(n ; \mathbf{R})$. In particular, $\operatorname{spec}(Q)=\operatorname{spec}(g(Q))$ if and only if $Q \in \mathbf{V}(\beta)$ for some bijection $\beta$ of $\mathbf{Z}^{n}$. We now consider the case that $\mathbf{V}(\beta) \neq \delta(n ; \mathbf{R})$ for all bijections $\beta$. It only remains to show that a neighborhood $U_{0}$ of $P$ exists with

$$
\left\{Q \in U_{0} \mid \operatorname{spec}(Q)=\operatorname{spec}(g(Q))\right\}=U_{0} \cap \bigcup_{n=1}^{t} \mathbf{v}\left(\beta_{n}\right)
$$


for appropriate bijections $\beta_{1}, \ldots, \beta_{t}$. Let $U_{0}$ (resp. $V_{0}$ ) be a relatively compact neighborhood of $P$ (resp. $S$ ) such that $\bar{U}_{0}, \bar{V}_{0} \subset \delta(n ; \mathbf{R})$ and $g\left(U_{0}\right) \subset V_{0}$. Now from $U_{0}, V_{0} \subset \delta(n ; \mathbf{R})$ we have $0<c_{1} \leqslant \lambda_{\min }(Q)$, $\lambda_{\max }(Q) \leqslant c_{2}$ for $Q \in U_{0}$ and $0<c_{3} \leqslant \lambda_{\min }(R), \lambda_{\max }(R) \leqslant c_{4}$ for $R \in V_{0}$. Let $\mathscr{B}$ be the set of all bijections of $\mathbf{Z}^{n}$. Trivially

$$
\left\{Q \in U_{0} \mid \operatorname{spec}(Q)=\operatorname{spec}(g(Q))\right\}=U_{0} \cap \bigcup_{\beta \in \mathscr{B}} \mathbf{V}(\beta) .
$$

Proceeding by contradiction we assume an infinite set $\left\{\beta_{\alpha}\right\}, \alpha \in \mathbb{Q}$, of bijections exists such that (i) $\left\{Q \in U_{0} \mid \operatorname{spec}(Q)=\operatorname{spec}(g(Q))\right\}=U_{0} \cap$ $\bigcup_{\alpha \in \mathbb{Q}} \mathbf{V}\left(\beta_{\alpha}\right)$, (ii) $\mathbf{V}\left(\beta_{\alpha}\right)$ is not properly contained in $\mathbf{V}(\beta), \beta \in \mathscr{B}$, and (iii) $\mathbf{V}\left(\beta_{\alpha}\right) \neq \mathbf{V}\left(\beta_{\alpha^{\prime}}\right)$ for $\alpha \neq \alpha^{\prime}$. Let $\left\{\beta_{l}\right\}$ be a sequence chosen from $\left\{\beta_{\alpha}\right\}$, $\alpha \in \mathbb{Q}$. Given $Q_{l} \in U_{0} \cap \mathbf{V}\left(\beta_{l}\right)$, then

$$
c_{1}\left\|\beta_{l}(N)\right\|^{2} \leqslant Q_{l}\left[\beta_{l}(N)\right]=g\left(Q_{l}\right)[N] \leqslant c_{4}\|N\|^{2} .
$$

In particular, for each $N \in \mathbf{Z}^{n}$ there are at most finitely many possibilities for $\beta_{l}(N)$. By Cantor diagonalization we obtain a subsequence $\left\{\beta_{p}\right\}$ such that for each $N \in \mathbf{Z}^{n}, \beta_{p}(N)$ is independent of $p$ for $p$ sufficiently large. Now we define $\beta_{\infty}(N)=\lim _{p \rightarrow \infty} \beta_{p}(N)$ for each $N \in \mathbf{Z}^{n}$. $\beta_{\infty}$ is an injection of $\mathbf{Z}^{n}$ into $\mathbf{Z}^{n}$. Specifically for $N \neq M \in \mathbf{Z}^{n}$ there is a $p_{0}$ and for $p>p_{0}, \beta_{\infty}(N)=$ $\beta_{p}(N) \neq \beta_{p}(M)=\beta_{\infty}(M)$. $\beta_{\infty}$ is a surjection of $\mathbf{Z}^{n}$ to $\mathbf{Z}^{n}$. Given $Q_{p} \in U_{0} \cap$ $\mathbf{V}\left(\beta_{p}\right)$ then

$$
c_{2}\left\|\beta_{p}(N)\right\|^{2} \geqslant Q_{l}\left[\beta_{p}(N)\right]=g\left(Q_{p}\right)[N] \geqslant c_{3}\|N\|^{2} .
$$

Fix $M_{0} \in \mathbf{Z}^{n}$; then $M_{0}=\beta_{p}\left(\beta_{p}^{-1}\left(M_{0}\right)\right)$ and thus $c_{2} / c_{3}\left\|M_{0}\right\|^{2}>\left\|\beta_{p}^{-1}\left(M_{0}\right)\right\|^{2}$. There is a $p_{1}$ and for $p \geqslant p_{1}, \beta_{p}(N)=\beta_{\infty}(N)$ for $N$ such that $\|N\|^{2}<$ $c_{2} / c_{3}\left\|M_{0}\right\|^{2}$. In particular, for $p \geqslant p_{1}$,

$$
M_{0}=\beta_{p}\left(\beta_{p}^{-1}\left(M_{0}\right)\right)=\beta_{\infty}\left(\beta_{p}^{-1}\left(M_{0}\right)\right) .
$$

The set $\left\{Q \in \mathbf{R}^{m} \mid Q\left[\beta_{\infty}(N)\right]=g(Q)[N], N \in \mathbf{Z}^{n}\right\}$ is a subspace of $\mathbf{R}^{m}$. Thus a constant $c_{5}>0$ exists with $\mathbf{V}\left(\beta_{\infty}\right)=\left\{Q \in \delta(n ; \mathbf{R}) \mid Q\left[\beta_{\infty}(N)\right]=\right.$ $\left.g(Q)[N],\|N\|<c_{5}\right\}$. For an appropriate $p_{2}, \beta_{p}(N)=\beta_{\infty}(N)$ for $p \geqslant p_{2}$ and $\|N\|<c_{s}$. In particular, $\mathbf{V}\left(\beta_{p}\right) \subset \mathbf{V}\left(\beta_{\infty}\right), p \geqslant p_{2}$. The containment $\mathbf{V}\left(\beta_{p}\right) \subset$ $\mathbf{V}\left(\beta_{\infty}\right)$ is not proper by the maximality condition for the $\mathbf{V}\left(\beta_{\alpha}\right), \alpha \in \mathbb{Q}$. Thus $\mathbf{V}\left(\beta_{p}\right)=\mathbf{V}\left(\beta_{\infty}\right)$ for $p \geqslant p_{2}$, a contradiction. The proof is complete.

Proof OF TheOREM 3. Let $g$ be a map defined by $g(Q)=R$ where $Q\left[M_{k}\right]=R\left[e_{k}\right], 1<k \leqslant n$ and $Q\left[M_{i j}\right]=R\left[e_{i j}\right], 1 \leqslant i<j \leqslant n$. Let $\beta$ be a bijection of $\mathbf{Z}^{n}$ such that

$$
Q[\beta(N)]=g(Q)[N] \text { for all } N \in \mathbf{Z}^{n}
$$

and all $Q$ in an open set $U$. The map $g$ is defined and (1) holds throughout $\mathbf{R}^{m}$. We deduce from $\operatorname{spec}(Q)=\operatorname{spec}(g(Q))$ for all $Q \in \delta(n ; \mathbf{R})$ that $g(Q)$ $\in \delta(n ; \mathbf{R})$ for all $Q \in \mathcal{S}(n ; \mathbf{R})$. The fibers $g^{-1}(g(Q)), Q \in \mathcal{S}(n ; \mathbf{R})$ are 
finite from Theorem 2. It now follows that $g$ is a linear isomorphism of $\mathbf{R}^{m}$. Trivially the equations $g^{-1}(R)[N]=R\left[\beta^{-1}(N)\right]$ for all $N \in \mathbf{Z}^{n}$, all $R \in \mathbf{R}^{m}$ hold; $g^{-1}$ maps $\delta(n ; \mathbf{R})$ into $\delta(n ; \mathbf{R})$. Define $G_{n}$ to be the group of all linear isomorphisms $g$ of $\mathbf{R}^{m}$ for which there is a $\beta$ and (1) holds. Referring to Lemmas 4 and 5 the proof is complete.

Definition 6. A vector $N \in \mathbf{Z}^{n}$ is primitive if $N \neq p M$ for $M \in \mathbf{Z}^{n}$ and $p \in \mathbf{Z}-\{0, \pm 1\}$.

THEOREM 7. $G_{n}$ coincides with the transformation group induced by $\mathrm{GL}(n ; \mathrm{Z})$.

Proof. If $Q\left[N_{0}\right]$ is the smallest positive value in the spectrum of $Q$ then $N_{0}$ is primitive. Remove the sequence $\left\{p^{2} Q\left[N_{0}\right]_{p=1}^{\infty}\right\}$ from the spectrum of $Q$. The smallest remaining positive value $Q\left[N_{1}\right]$ corresponds to a primitive vector $N_{1}$; remove the sequence $\left\{p^{2} Q\left[N_{1}\right]\right\}_{p=1}^{\infty}$. Continuing in this manner all primitive vectors are identified, and for $g$ and $\beta$ satisfying (1), $\beta$ preserves this construction.

We consider $g \in G_{n}$ and show that $g$ can be transformed to the identity by conjugation with elements of $\mathrm{GL}(n ; \mathbf{Z})$. Let $g$ be defined by the equations $Q\left[M_{k}\right]=g(Q)\left[e_{k}\right]$ and $Q\left[M_{i j}\right]=g(Q)\left[e_{i j}\right] . M_{n}$ is a primitive vector; thus T $\in \mathrm{GL}(n ; \mathrm{Z})$ exists with $\Re e_{n}=M_{n}$. Replacing $g$ with the map $Q \rightarrow$ $g\left(Q\left[\Re^{-1}\right]\right)$ we can assume $M_{n}=e_{n}$. We now proceed by induction on the dimension $n$. For $n=2$ it is classical that the eigenvalue spectrum determines the tori in $O(2) \backslash \mathrm{GL}(2 ; \mathbf{R}) / \mathrm{GL}(2 ; \mathrm{Z})[1]$, [6]. Define $\Phi$ to be the projection of $\mathbf{R}^{n}$ onto the first $n-1$ coordinates. Let $\Psi$ be the natural inclusion of $\mathbf{R}^{n-1}$ into $\mathbf{R}^{n}$ with image the first $n-1$ coordinates of $\mathbf{R}^{n}$. Given $Q$ a symmetric quadratic form on $\mathbf{R}^{n}$, define $\tilde{Q}$ a symmetric quadratic form on $\mathbf{R}^{n-1}$ by $\tilde{Q}[x]=Q[\Psi(x)]$ for $x \in \mathbf{R}^{n-1}$. Let $Q_{s}$ be a curve from $[0,1]$ into $\mathbf{R}^{m}$ such that (i) $Q_{s} \in \mathcal{S}(n ; \mathbf{R})$ for $0<s<1$, (ii) $Q_{1}\left[e_{n}\right]=0$, and (ii) $\tilde{Q}_{1} \in \mathcal{S}(n+1$; R). We observe that $Q_{1}[\beta(N)]=g\left(Q_{1}\right)[N]$ for all $N \in \mathbf{Z}^{n}$; in particular, $g\left(Q_{1}\right)$ is positive semidefinite and $Q_{1}\left[M_{k}\right]=g\left(Q_{1}\right)\left[e_{k}\right]$ with $M_{n}=e_{n}$. For $R=\left(r_{i j}\right)$ positive semidefinite we have by the Cauchy Schwarz inequality that $r_{i j}^{2}<r_{i i} r_{j j}$; in particular, $e_{i}^{t} Q_{1} e_{n}=e_{i}^{t} g\left(Q_{1}\right) e_{n}=0,1<i<n$. Assume that the entries $q_{i j}$ of $Q_{1}$ with $1<i<j<n-1$ are rationally independent. For $N, M \in \mathrm{Z}^{n-1}$ with $\tilde{Q}_{1}[N]=\tilde{Q}_{1}[M]$ it follows that $N= \pm M$. We observe for $\gamma \neq 0$ in the spectrum of $Q_{1}$ that $\gamma$ has multiplicity two in the spectrum of $\tilde{Q}_{1}$. As $Q_{1}[\beta(N)]=g\left(Q_{1}\right)[N]$ for every $N \in \mathbf{Z}^{n}$ we conclude $\tilde{Q}_{1}$ and $\widetilde{g\left(Q_{1}\right)}$ have the same spectrum. The map $g$ induces a linear map $\tilde{g}$ from a neighborhood of $\tilde{Q}_{1} \in \mathcal{S}(n-1 ; \mathbf{R}) \subset \mathbf{R}^{p}, p=n(n-1) / 2$, to a neighborhood of $\widetilde{g\left(Q_{1}\right)} \in$ $\delta(n-1 ; \mathbf{R})$. The map $\tilde{g}$ preserves the spectrum with the possible exception of the forms with rationally dependent entries. Those forms in $\delta(n-1 ; \mathbf{R})$ with rationally dependent entries form a subset of measure zero. Referring to Theorem 3 and Lemmas 4 and 5 we conclude $\tilde{g}$ induces a spectrum preserving isomorphism of $\delta(n-1 ; \mathbf{R})$ to $\delta(n-1 ; \mathbf{R})$. 
The map $\tilde{g}$ by the induction hypothesis corresponds to a $\mathscr{Z} \in \mathrm{GL}(n-1$; Z). Define $\mathscr{I}_{1}=\left(\begin{array}{c}\mathscr{Z} \\ 0\end{array}\right), R=h(Q)=g(Q)\left[\mathscr{E}_{1}^{-1}\right]$ and $\alpha(N)=\beta\left(\mathscr{L}_{1}^{-1} N\right)$. We observe that $\alpha$ is a bijection of $\mathbf{Z}^{n}$ with $h(Q)[N]=Q[\alpha(N)]$. It follows from the induction hypothesis that $\Phi(\alpha(N))= \pm \Phi(N)$; for our purposes we can assume $\Phi(\alpha(N))=\Phi(N)$. We have $\alpha\left(e_{n}\right)=e_{n}$ from the definition of $\alpha$ and the fact that $\beta\left(e_{n}\right)=e_{n}$. A matrix $\theta$ is now defined by the equations $\theta e_{k}=\alpha\left(e_{k}\right), 1<k<n$. It is clear that $\theta$ has integer entries and that $\operatorname{det}(\theta)=1$. We conclude that $\theta \in G L(n ; Z)$. Define the map $f \in G_{n}$ by $R=f(Q)=h\left(Q\left[\theta^{-1}\right]\right)$ and the bijection $\delta$ of $\mathbf{Z}^{n}$ by $\delta(N)=\theta^{-1} \alpha(N)$ for all $N \in \mathbf{Z}^{n}$. The map $f$ is also defined by the equations $R\left[e_{k}\right]=Q\left[\theta^{-1} \alpha\left(e_{k}\right)\right]$ and $R\left[e_{i j}\right]=Q\left[\theta^{-1} \alpha\left(e_{i j}\right)\right]$. We conclude that $\Phi(\delta(N))=\Phi(N)$ for all $N \in$ $\mathbf{Z}^{n}$ from the definition of $\theta$ and the corresponding fact for $\alpha$. The map $f$ modulo a choice of signs will be the identity in $G_{n}$. Noting that $\delta\left(e_{k}\right)=e_{k}$, $1<k<n$, we conclude for $R=f(Q)$ with $R=\left(r_{i j}\right)$ and $Q=\left(q_{i j}\right)$ that $r_{k k}=q_{k k}, 1<k<n$. Now consider a particular entry $r_{i j}, 1<i<j<n$, and the defining equation

$$
r_{i j}=\left(Q\left[\delta\left(e_{i}+e_{j}\right)\right]-Q\left[e_{i}\right]-Q\left[e_{j}\right]\right) / 2 .
$$

We note from $\Phi(\delta(N))=\Phi(N)$ for $N \in \mathbf{Z}^{n}$ that $\delta\left(e_{i}+e_{j}\right)=e_{i}+e_{j}+s_{i j} e_{n}$, $s_{i j} \in \mathbf{Z}$. The defining equation for $r_{i j}$ becomes

$$
r_{i j}=q_{i j}+s_{i j} q_{i n}+s_{i j} q_{j n}+s_{i j}^{2} q_{n n} / 2 \text {. }
$$

A short computation shows that $r_{i j}$ is independent of $q_{n n}$ if and only if $s_{i j}=0$ for $j<n$ or $s_{i j}=0,-2$ for $j=n$. Now consider $Q \in \delta(n ; \mathbf{R})$ to be diagonal with $q_{k k}, k<n$, fixed. Assume $r_{i j}$ depends on $q_{n n}$; $r_{i j}^{2}$ thus has quadratic growth in $q_{n n}$ for $q_{n n} \rightarrow \infty$. Considering the inequality $r_{i j}^{2} \leqslant r_{i i} r_{j j}=$ $q_{i i} q_{j j}$ we have a contradiction since $i<n$ and $q_{i i}$ is fixed for $q_{n n} \rightarrow \infty$. We conclude $r_{i j}=q_{i j}$ for $1<i, j \leqslant n-1, r_{i n}= \pm q_{i n}, 1<i<n-1$ and $r_{n n}=$ $q_{n n}$. Now to ascertain the signs choose $Q \in \delta(n ; \mathbf{R})$ with rationally independent entries. Assume there exist $i, k<n$ with $q_{k n}=r_{k n}$ and $q_{i n}=-r_{i n}$, as otherwise $R=Q[\mathcal{Y}]$ where

$$
\text { Y }=\left(\begin{array}{cc}
\mathrm{id}_{n-1} & 0 \\
0 & \pm 1
\end{array}\right)
$$

and $\mathrm{id}_{n-1}$ is the identity in $\mathrm{GL}(n-1 ; \mathrm{Z})$. For $Q=\left(q_{a b}\right)$ and $R=\left(r_{a b}\right)=$ $f(Q)$ there exists a vector $M=\left(m_{1}, \ldots, m_{n}\right)^{t}$ such that $R[M]=Q\left[e_{k}+e_{i}\right.$ $\left.+e_{n}\right]$. By the rational independence we have $q_{n n}=m_{n}^{2} r_{n n}, q_{k i}=m_{k} m_{i} r_{k i}$, $q_{i n}=m_{i} m_{n} r_{i n}$ and $q_{k n}=m_{k} m_{n} r_{k n}$. From the definition of $f$ we have $q_{n n}=r_{n n}$ and $q_{k i}=r_{k i}$; thus $m_{k} m_{i}=m_{n}^{2}=1$. By assumption $m_{k} m_{n}=1$ and $m_{i} m_{n}=$ -1 ; combining these relations $1=m_{k} m_{i}=m_{k} m_{n} m_{n} m_{i}=-1$, a contradiction. The proof is now complete.

Theorems 3 and 7 are combined in the following. 
THEOREM 8. Isospectral tori $T_{0}=\mathbf{R}^{n} / A_{0} \mathbf{Z}^{n}, T_{1}=\mathbf{R}^{n} / A_{I} \mathbf{Z}^{n}$ are isometric if and only if at least one of the quadratic forms $\left(A_{0}^{t} A_{0}\right),\left(A_{1}^{t} A_{1}\right)$ is an element of $\delta(n ; \mathbf{R})-\mathbf{V}_{n}$. If $T_{0}$ and $T_{1}$ are not isometric then the entries of the matrix $\left(A_{1}^{\prime} A_{1}\right)$ are linear combinations with rational coefficients of the entries of the matrix $\left(A_{0}^{t} A_{0}\right)$. The set $\mathbf{V}_{n}$ is $\delta(n ; \mathbf{R})$ intersected with a countable union of subspaces of $\mathbf{R}^{m}$; these subspaces are defined by equations with rational coefficients.

COROLlaRY 9. Let $\mathbf{R}^{n} / A \mathbf{Z}^{n}$ be given such that the entries of the form $\left(A^{\prime} A\right) \in \mathrm{V}_{n} \subset \delta(n ; \mathbf{R})$ satisfy at most $p$ distinct linear homogeneous equations with rational coefficients. The form $\left(A^{A} A\right)$ is contained in a subspace $W$, with $W \cap \delta(n ; \mathbf{R}) \subset \mathbf{V}_{n}$ and $m-p<\operatorname{dim} W<m-1$. If the entries of the form are rationally independent $\mathbf{R}^{n} / A \mathbf{Z}^{n}$ is uniquely determined by its eigenvalue spectrum.

A form $Q \in \delta(n ; \mathbf{R})$ is called semi-integral if for $Q=\left(q_{i j}\right), q_{k k} \in \mathbf{Z}$, $1<k<n$ and $2 q_{i j} \in \mathrm{Z}, 1<i<j<n . Q$ semi-integral is equivalent to the statement $\operatorname{spec}(Q) \subset \mathbf{Z}$. The semi-integral forms are of particular number theoretic interest.

LEMMA 10. Let $\mathbf{V}_{n}$ be nonempty for a particular $n$. Then semi-integral forms $Q_{0}, Q_{1} \in \mathbf{V}_{n}$ exist.

Proof. $\mathbf{V}_{n}$ is nonempty by hypothesis. Observe that rational points are dense in subspaces defined by rational equations. In particular, $P_{0}, P_{1} \in \mathrm{V}_{n}$ exist with $\operatorname{spec}\left(P_{0}\right)=\operatorname{spec}\left(P_{1}\right)$ and $P_{0}$ has rational coordinates. Since $P_{0}$ is rational a positive integer $p$ exists with $p P_{0}$ semi-integral and thus $p \operatorname{spec}\left(P_{0}\right)$ $=p \operatorname{spec}\left(P_{1}\right) \subset \mathbf{Z}$. In particular, $p P_{1}$ is semi-integral.

Previous results show that $V_{n}$ is nonempty for $n>12$ [1], [2]. In fact, an elementary construction shows that if $\mathbf{V}_{n}$ is nonempty then all $\mathbf{V}_{m}, m>n$, are nonempty. From Lemma 10 it suffices to consider the semi-integral forms in the cases $n=3, \ldots, 11$. We also note that a result analogous to Theorem 8 has been obtained for the case of compact Riemann surfaces [8], [9].

\section{REFERENCES}

1. M. Berger, P. Gauduchon and E. Mazet, Le spectre d'une varièté Riemannienne, SpringerVerlag, Berlin, 1971.

2. M. Berger, Geometry of the spectrum, Proc. Sympos. Pure Math., vol. 27, Part 2, Amer. Math. Soc., Providence, R. I., 1975, pp. 129-152.

3. J. W. S. Cassels, An introduction to the geometry of numbers, Springer-Verlag, Berlin, 1975, p. 135.

4. I. M. Gel'fand, M. I. Graev and I. I. Pyaetskii-Shapiro, Representation theory and automorphic functions, Saunders, Philadelphia, 1969.

5. R. C. Gunning, Lectures on modular forms, Ann. of Math. Studies, no. 48, Princeton Univ. Press, Princeton, N. J., 1962. 
6. H. P. McKean, Selberg's trace formula as applied to a compact Riemann surface, Comm. Pure Appl. Math. 25 (1972), 225-246.

7. J. Milnor, Eigenoalues of the Laplace operator on certain manifolds, Proc. Nat. Acad. Sci. U.S.A. 51 (1964), 542.

8. S. Wolpert, The eigenoalue spectrum as moduli for compact Riemann surfaces, Bull. Amer. Math. Soc. 83 (1977), 1306-1308.

9. _ـ The length spectrum as modult for compact Riemanur surfaces (to appear).

Departuent of Mathematics, University of Maryland, Collbob Park, Maryland 20742 